\title{
An annotation protocol for evaluative stance in discourse
}

\author{
Laura Hidalgo Downing", Paula Pérez-Sobrino², Laura Filardo-Llamas ${ }^{3}$, Carmen Maíz-Arévalo ${ }^{4}$, \\ Begoña Núnez-Perucha ${ }^{4}$, Alfonso Sánchez-Moya $a^{4}$,Julia T. Williams Camus ${ }^{5}$
}

${ }^{1}$ Universidad Autónoma de Madrid, Spain

2Universidad de La Rioja, Spain

${ }^{3}$ Universidad de Valladolid, Spain

${ }^{4}$ Universidad Complutense de Madrid, Spain

${ }^{5}$ Universidad de Cantabria, Spain

Corresponding author: Laura Hidalgo Downing, laura.bidalgo@uam.es

This paper is part of the work carried out in the funded research project Stance and subjectivity in discourse: towards an integrated model of the analysis of epistemicity, effectivity, evaluation and intersubjectivity from a critical discourse perspective (PGC2018-095798-B-I00).

In this paper we propose a protocol for the annotation of evaluative stance across discourse types. We have used the protocol to annotate four 100,000-word corpora in English: opinion articles (The Guardian and The Times), science popularization in the press (The Guardian and The Times), political discourse (speeches delivered by British politicians) and fora on social issues (REDDIT).

The development of the protocol has gone through two main stages. The first stage has consisted in a preliminary theoretical definition of the model of evaluative stance and its main categories, drawing from research on stance, evaluation and critical discourse analysis, together with methods for the identification of metaphoricity (du Bois 2007, Martin and White 2005, Pragglejazz 2007, van Leeuwen 2008, Wodak and Meyer 2015, among others). The preliminary model was tested in samples of the corpora and subsequently, the protocol underwent a first initial refinement and revision.

The second stage has consisted in a process of establishing a good degree of inter-rater reliability for the full annotation of the corpora. The procedure of inter-rater reliability was carried out by three researchers (Hidalgo-Downing, Pérez-Sobrino, and Williams-Camus) who individually annotated samples from the corpora in four subsequent rounds. A joint discussion followed each round to discuss conflicting annotations and to refine the protocol for the ensuing round. The goal of these series of annotations was to know whether there was any variation in the inter-rater reliability with which evaluative stance was identified across researchers, rounds and genres. The results of the inter-rater reliability tests show a consistent increase in the kappa scores for the value category (positive vs negative evaluation) and, to a lesser extent, for metaphoricity (although, in both cases, kappa scores showed moderate to high agreement). These rounds were complemented with two rounds of annotation of sample texts by the full team (all seven researchers participating in this project) in order to ensure the understanding and uniform application of the criteria of the protocol for the annotation of the whole corpora.

\section{Contents}

0. Definitions of basic concepts 
1. Level 1: IDENTIFICATION OF EVALUATIVE STANCE (by PoS and function)

2. LEVEL 2: IDENTIFICATION OF EVALUATIVE METAPHOR

3. LEVEL 3: IDENTIFICATION OF VALUE (POSITIVE OR NEGATIVE)

\section{Definitions of basic concepts}

\section{Overview and definition of Evaluative stance}

In our model, Evaluative stance is a social act which expresses a positioning of a speaker/writer towards a specific topic, entity, social actor or event; by means of this positioning, the speaker/writer aligns or disaligns with real or potential addressees and communities of speakers according to shared systems of beliefs, expressing a positive or negative value towards the topic, entity or event. Evaluative stance as a social act is realised in discourse by means of specific stance choices of lexical evaluative expressions. These lexical evaluative expressions may express an assessment of words or phrases and may (1) classify entities or events according to social systems of beliefs - we have termed this type classiffing, and corresponds to the prototypical function of evaluative nouns and verbs, or (2) qualify entities or events according to social systems of belief - we have termed this type predicational, and corresponds to the prototypical function of adjectives. Lexical evaluative expressions may also express an attitude or opinion about a whole proposition - we have termed this type attitude; this function is typically performed by sentential adverbs. Classifying and predicational functions can be non-metaphoric or metaphoric. Classifying, predicational and attitude functions may express either inscribed (explicit) or evoked (implicit) positive or negative value. Lexical markers of stance may be amplified or mitigated by means of intensifiers and downtoners, which we have called Graduation markers, adapting some of the categories within Appraisal Theory.

The expression identified as expressing evaluative stance must meet the three following conditions (following the definition of stance in Du Bois 2007) ${ }^{1}$. It must:

$\square$ Evaluate entities, events or propositions yielding positive or negative evaluations. In case of doubt regarding what counts as evaluative expression, check Martin and White (2005).

$\square$ Express a positioning of the speaker/writer towards the topic of the article . It should be recalled here that we do not annotate epistemic (certainty, possibility, probability) and/or effective (obligation, necessity, volition) stance. E.g.: clearly, obviously, maybe, perhaps, likely, possibly, it is possible, it is clear/obvious/evident, I am sure, it is necessary, there is an obligation, there is a necessity; I lean towards, I object...

$\square$ Express alignment or disalignment of the writer/speaker with communities of speakers (racist vs anti-racist, in favour of conservative ideas, against conservative ideas, neither for nor against conservative ideas, a criticism of a position, a positive review of a position, etc.)

$\square$ What does this mean (in practical terms)? The author must (1) express an attitude towards or belief about a topic, a social actor or event (not describe!) and (2) show agreement / adherence/support (alignment) or distance/disagreement (disalignment) towards an expressed or evoked position. We code the evaluation as positive or negative with respect to the author's positioning, not towards a

${ }^{1}$ Du Bois, J. W. 2007. The Stance Triangle. In Englebretson, R. (Ed.) Stancetaking in Discourse. John Benjamins, Amsterdam, pp. 139-182. 
potential addressee's possible position! alignment $=$ positive; disalignment $=$ negative. See Figure 1.

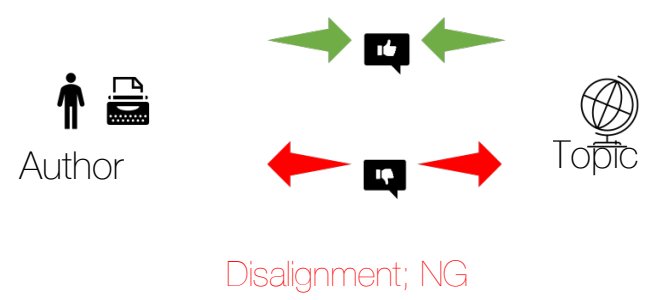

Figure 1. understanding of evaluative stance as author's alignment (positive evaluation) or disalignemnt (negative evalution) towards a topic.

\section{Cases where we $\mathrm{DO}$ annotate words as evaluative}

*** Note that words with inscribed evaluation. In this context, we understand 'inscribed' as words that have evaluative meaning by default (good, bad, etc.) and must always be annotated, EXCEPT when they are under the scope of a reporting verb (in which case we consider this second-degree stance).

Cases where we DON'T annotate words as evaluative

*** Note that some words are descriptive and not evaluative. Some words which express difference or contrast (different, opposite, contrary, distinct) represent a reality that is divided according to some feature. However, the use of the word does not necessarily mean that the speaker/writer is expressing a positioning and alignment towards one of the factions.

*** Note that some descriptive words are part of the jargon of a specific genre and are not evaluative. Examples:

- Politics: deal, serve.

- Science: decline, harm, risk, vulnerability.

\section{In case of DOUBT:}

*** In case of doubt whether an expression is evaluative or not, we will look the word up in the following two dictionaries of reference:

- MacMillan Dictionary: https://www.macmillandictionary.com/

- Collins Cobuild Dictionary: https://www.collinsdictionary.com/dictionary/english

In order for the word/expression to be annotated as evaluative, the definition in one of these dictionaries must include an evaluative connotation. For example: a deal that works.

- Look up the definition of 'work' (Collins Cobuild, lexical entry 12): "If an idea, system, or way of doing something works, it is successful, effective, or satisfactory."

- This definition includes three evaluative terms (successful, effective, or satisfactory) which make explicit the connotation of the word, and 
therefore can be considered to have 'inscribed' evaluation (it should be coded according to this protocol).

\section{Other practicalities:}

*** Note that, for the sake of practicality, we restrict the annotation of evaluative expressions to grammatical categories (NP, AP, ADVP, VP, but not PP).

***Note that numerous expressions require that you annotate together both the head of the phrase and its modifier as evaluative expression, especially in the function CLA, but also in PRE and ATT: casual bigotry (CLA), white nationalism (CLA), socially acceptable (PRE), however big or small (PRE), (for) the better (PRE). We annotate these as one evaluative expression, not two. The criterion is whether there is unity of meaning between both lexical items.

\section{LEVEL 1: IDENTIFICATION OF EVALUATIVE STANCE (by PoS and function)}

\section{PoS: Is the evaluative expression a phrase (NP, AP, ADVP, VP) that conveys positive or negative evaluation?}

(Reminder: we annotate only $1^{\text {st }}$ degree stance. There must be a reporting verb or expression indicating attribution (according to $X$, In $X$ 's words...) to consider the positioning $2^{\text {nd }}$ degree stance - in absence of reporting verb, treat it as $1^{\text {st }}$ degree.)

If the expression is not evaluative: disregard

If the expression is evaluative: annotate function

\section{Function:}

\section{If Noun phrase (NP)}

Code it as classifier (CLA) if it is a head in a NP (that is, it does not modify another word). Eg: Trump is a demagogue; Brexit is a disaster; Islamic terrorism, the far right, right-wing pundits, hate speech, white nationalism, the truth.

- Annotate social categories: terrorism, nationalism...

- Annotate full lexical units: Islamist terrorism, white nationalism, far right...

*** Specific for Politics: we annotate expressions such as enormous potential, leading the way, improving... because they're used in this context to project positive evaluation (but perhaps they are not coded in other genres).

***Note on antithesis: when encounter antithesis (evaluative terms in a relation of opposition in discourse), we annotate both the negative evaluation and the positive evaluation, even if one of the evaluative terms is only introduced in order to contrast with the other. E.g. When I was born ... casual bigotry (EV, NP, CLA, NMT, NG) was a socially acceptable fact (EV, NP, CLA, NMT, PS) of daily life.

*** Words such as truth/lies: Annotate as CLA whenever they express a position towards the information as reliable or not. That is, whether something is true or false can be a subjective evaluation (which we annotate) or an objective observation 
(which we do not annotate). Notice that the evaluative use appears often in politics and press in the context of the topic of fake news.

*** Note that some nouns can modify as if they were adjectives, as in this is a key step in the research. Key modifies step, but it is a noun. However, its function will be PREDICATIONAL. Code as PREDICATIONAL if evaluative.

If Adjectival phrase (AP):

PRE: if the AP expresses a quality or property of a social actor or event relevant to the topic of the article, based on socio-cultural values and beliefs, typically with positive and negative evaluation. E.g.: Trump is the worst president of the US to date; Brexit is a painful and difficult process

- Include adjectives denoting fairness (fair, understandable...) because they imply agreement/support (alignment).

- Include personal interpretations/reformulations of technical data: a major study; serious bealth problems; her self-confidence was at a very low level. (note here that we annotate the modifier "very" as GRA - Graduation!).

- Examples such as this is true involve evaluation if there is a division of opinions about the statement (e.g in context of fake news).

- If the AP is preceded by 'not' (or sometimes ' $n o$ ), notice that the value is inverted. Example: He was not glamorous: The value of 'not glamorous' is negative, the contrary of glamorous.

A'T'T: if the AP forms part of an expression which provides a comment on the whole sentence/proposition. E.g.: I am sad/concerned to say this is the end; sad to say; it is notable that....; it is worth saying that...

- Do not code as AP words such as key step, where key is a Noun.

- $\quad$-ed participles. Distinction between Adjective vs Verb depends on the structure of the sentence.

- If it is a structure of identification (X is $\mathrm{Y}$ ): I am a happy/lost/ bungry/excited/ overwhelmed/decided/ indignant/determined teacher etc. it will not be a verb. It will be Noun or Adjective.

- When the -ed participle follows the verb 'to be': If there is a passive (Labour is represented by....), the item following the verb to be is a full verb.

- When the -ed participle follows other verbs, it can only be a verb (I have relinquished all hope, We never abandoned hope).

- -ing participles. Distinction between Adjective vs Verb depends on the structure of the sentence.

- It is an adjective when it premodifies a noun: glittering career, worrying news. It is a noun when it functions as subject of a clause: Worrying now is now use. It is a verb when it indicates continuous tense of a verb (I was craving for sugar, they bad been crying for help).

If Adverbial phrase (ADVP):

GRA: adverbs that modify AP to express intensity, amplification or are downtoners as: peculiarly painful, terribly mistaken, dramatically different, very true, true love, little bope.

- Prototypical markers of Graduation are amplifiers (very, terribly) or downtoners (not much, not very). Some Graduation markers express 
quantification (little/much evidence). Notice that this is one of the features that distinguishes Graduation form the Predicational function, since the Predicational function will not include markers that are quantifiers. Quantifiers as a grammatical category are Determiners, not Adjectives. Some other markers of Graduation may express what Appraisal calls a sharpening focus, as in real problem.

- Graduation expressions are not annotated in terms of positive or negative value, but in terms of more (MOR) or less (LES), which will refer to whether the expression amplifies or reduces the strength of the expression if modifies, thus expressing the degree of alignment or disalingment of the writer/speaker towards the evaluative expression.

- Note that the distinction between adverbs that have GRA, PRE and AT'T has to do with the scope of the adverbs, that is: what do they express an attitude to?

- GRA (terribly happy) take scope over phrases

- GRA take scope over adjectives, adverbs or APs or AdvPs (peculiarly painful)

PRE: if the adverb expresses a manner of doing the action expressed in a VP (that is, it only modifies the following verb or VP as manner adverb). E.g.: (clumsily collected votes) or a quality of an AP (Labour is unambiguoushy opposed to...). In this second sentence, 'opposed' is adjective. See note on this above.

- Note that the distinction between adverbs that have GRA, PRE and AT'T has to do with the scope of the adverbs, that is: what do they express an attitude to?

- PRE (clumsily collected...) takes scope over phrases, AT'T take scope over clauses or sentences.

- PRE take scope over the VP or Vb (clumsily walked in)

AT'T if the adverb expresses a comment on the whole sentence/proposition (affects whole statement). E.g.: Luckily, elections will be soon.

Do not annotate Adverbs such as indeed or actually as ATT. These are not stance adverbs (for doubts on what is a stance adverb, see the Longman Grammar of English, Biber et al. 1999), specifically Disjuncts.

If Verb Phrase (VP):

CLA: if the VP represents an event relevant to the topic of the article, typically with positive and negative evaluation. E.g.: Labour wants to leave the EU but to close the door nicely, improve, recoiled.

***Don't code Prepositional Phrases (PP).

\section{LEVEL 2: IDENTIFICATION OF EVALUATIVE METAPHOR}

Basic criterion for evaluative metaphors: Does the expression consist of a mapping of a source domain with a positive or negative value mapped onto a target domain? 
$\square$ If yes: annotate as MT. E.g.: their glittering careers, if you erode the natural capital, enlightened environmental legislation, a strong economy needs a bealthy environment.

$\square$ If no: annotate as NMT. E.g.: successful careers

○ AP and ADVP that function as ATT are usually NMT (usually, not always! eep an eye open).

Remember that not all metaphors are evaluative! Examples of non-evaluative metaphors: some quarters of the Christian community, entering the toughest phase of negotiations, keep trade friction-free. Also, these expressions are highly entrenched or conventionalized metaphors.

Don't code very conventional metaphors that are part of stock phrases such as "far right", "white nationalism".

Consider the following examples, which are similar because they belong to the same extended metaphor IDEOLOGY IS A JOURNEY/LANDSCAPE:

Context: the writer is drawing a comparison between Islamist terrorists and gun supporters.

\begin{tabular}{|l|l|}
\hline $\begin{array}{l}\text { X "travelled the exact same route as the } \\
\text { Islamist forebears" }\end{array}$ & $\begin{array}{l}\mathbf{X} \text { is "exiled in the boondocks" (of the } \\
\text { web) }\end{array}$ \\
\hline $\begin{array}{l}\text { It looks like evaluative, because it is a } \\
\text { periphrasis of "radicalized" (which would } \\
\text { be annotated as evaluative). }\end{array}$ & $\begin{array}{l}\text { Exile" implies that there is a reason why a } \\
\text { person must leave a place (usually bad } \\
\text { news), and therefore we assume there is } \\
\text { disaligment. }\end{array}$ \\
$\begin{array}{l}\text { But "travelling" doesn't express any } \\
\text { alignment/disalignment of the writer by } \\
\text { itself (it's not positive or negative per se). } \\
\text { moved to Y place", which would be then like } \\
\text { the "travelling" example. }\end{array}$ \\
$\begin{array}{l}\text { DON'T ANNOTATE IT. } \\
\text { ANNOTATE IT. }\end{array}$ \\
\hline
\end{tabular}

IN CASE OF DOUBT

O Check for metaphoricity in a dictionary whenever you have the slightest doubt. Use the MacMillan Dictionary. If the metaphoric use of a word is listed as one of the basic entries of the dictionary, we will not annotate it.

\section{DON'T ANNOTATE}

O Examples of terms that are not metaphoric: the words key and vital. If the definition appears in lexical entry do not annotate (there may be exceptions to this). Definitions (Macmillan dictionary):

- Key: lexical entry 2: the thing that will do most to help you to achieve something.

- Vital: definition in Macmillan dictionary. Vital, adjective: very important, necessary or essential.

- Do not code highly conventionalized metaphors (again check for degree of metaphoricity in dictionaries). Example: I'm gutted that Northumbrian is now banned. Definition of gutted in Cambridge English dictionary: 'extremely disappointed and unhappy'. Not metaphoric.

- Other examples of non-evaluative metaphor: quarters of the Christian community, entering the toughest phase of negotiations, keep trade friction-free 
- Extended metaphor. In some texts we may find that metaphoric expressions cluster in a part of the text, creating an extended metaphor. On these occasions, an initial metaphor opens an evaluative frame which is then enriched with following terms. There are difficulties in deciding how many of the possible terms to annotate.

- Example: 'The critically ill NHS needs a big cash injection.' We annotate both ill and cash injection as metaphoric, since they form part of an extended metaphor related to illness.

\section{LEVEL 3: IDENTIFICATION OF VALUE (POSITIVE OR NEGATIVE)}

\section{Does the expression have a negative or positive value?}

If yes: annotate as PS (alignment) or NG (disalignment)

- Note that positive and negative evaluation is performed by a speaker/writer towards an object/entity/event mentioned or evoked in the discourse. It is not the evaluation the analyst assigns to that object/entity/event, it is not the value that a potential addressee may assign and it is not necessarily the value that object/entity/event may have in society at this moment in time.

If in doubt regarding the value of a term, check a dictionary for clues (Oxford, Cambridge and Collins Cobuild). Remember that some terms have inscribed (explicit) evaluation: good, bad, right, wrong, true, lie, biased, problem, potential.

\section{References}

Du Bois (2007) The Stance Triangle. In R. Englebretson (Ed.), Stancetaking in Discourse: Subjectivity, Evaluation, Interaction (pp. 139-182). Amsterdam: John Benjamins Publishing Company.

Hidalgo Downing, Laura (2020) Theme Specific panel on Evaluative stance and metaphor. Stance, intersubjectivity and identity International Conference. Universidad Complutense de Madrid, 911 September.

Hidalgo-Downing, L.; Filardo-Llamas, Pérez-Sobrino, P. \& Williams-Camus, J. (2019) "Evaluation across discourse domains. Metaphorical uses in advertising, fora and scientific discourses." Paper presented at the 43rd AEDEAN Conference held at the University of Alicante. November 13.

Marín Arrese1, Juana I., Laura Hidalgo Downing², Marta Carretero', Elena Domínguez Romero ${ }^{1}$, Sergio Ferrer Navas ${ }^{1}$, Laura Filardo Llamas ${ }^{5}$, Carmen Maíz Arévalo ${ }^{1}$, Victoria Martín de la Rosa ${ }^{1}$, Natalia Mora López ${ }^{1}$, Begoña Núñez Perucha ${ }^{1}$, Paula Pérez Sobrino ${ }^{4}$, Alfonso Sánchez Moya ${ }^{6}$, Julia Williams Camus ${ }^{3}$ (2019) Stancetaking in Discourses: Epistemicity, Effectivity, Evaluation. Actas del 43 Congreso AEDEAN.

Martin, J.R. and White, P.R.R. (2005) The Language of Evaluation: Appraisal in English. London: Palgrave McMillan.

Pragglejazz Group (2007) MIP: A Method for Identifying Metaphorically Used Words in Discourse. Metpahor and Symbol, 22(1), 1-39.

van Leeuwen, T. (2008) Discourse and Practice: New Tools for Critical Discourse Analysis. Oxford: Oxford University Press. 
Wodak, R. and M. Meyer (2015) Critical discourse studies: history, agenda, theory and methodology. In Wodak, Ruth and Michael Meyer (Eds.) Methods of Critical Discourse Studies Edition: 3rd revised edition. (pp. 2-21) London, Sage. 